\title{
On Certain Connected Resolving Parameters of Hypercube Networks*
}

\author{
Bharati Rajan, Albert William, Indra Rajasingh, S. Prabhu \\ Department of Mathematics, Loyola College, Chennai, India \\ Email: pravinovin@gmail.com
}

Received January 5, 2012; revised March 14, 2012; accepted March 21, 2012

\begin{abstract}
Given a graph $G=(V, E)$, a set $W \subset V$ is a resolving set if for each pair of distinct vertices $u, v \in V(G)$ there is a vertex $w \in W$ such that $d(u, w) \neq d(v, w)$. A resolving set containing a minimum number of vertices is called a minimum resolving set or a basis for $G$. The cardinality of a minimum resolving set is called the resolving number or dimension of $G$ and is denoted by $\operatorname{dim}(G)$. A resolving set $W$ is said to be a star resolving set if it induces a star, and a path resolving set if it induces a path. The minimum cardinality of these sets, denoted respectively by $\operatorname{sr}(G)$ and $\operatorname{pr}(G)$, are called the star resolving number and path resolving number. In this paper we investigate these resolving parameters for the hypercube networks.
\end{abstract}

Keywords: Resolving Set; Basis; Path Resolving Set; Star Resolving Set; Hypercube Network

\section{Introduction}

A query at a vertex $v$ discovers or verifies all edges and non-edges whose endpoints have different distance from $v$. In the network verification problem [1], the graph is known in advance and the goal is to compute a minimum number of queries that verify all edges and non-edges. This problem has previously been studied as the problem of placing landmarks in graphs or determining the metric dimension of a graph [2]. Thus, a graph-theoretic interpretation of this problem is to provide representations for the vertices of a graph in such a way that distinct vertices have distinct representations. This is the subject of the papers [3-5].

For an ordered set $W=\left\{w_{1}, w_{2} \cdots w_{k}\right\}$ of vertices and a vertex $v$ in a connected graph $G$, the code or representation of $v$ with respect to $W$ is the $k$-vector

$$
C_{W}(v)=\left(d\left(v, w_{1}\right), d\left(v, w_{2}\right) \cdots d\left(v, w_{k}\right)\right)
$$

where $d(x, y)$ is the distance between the vertices $x$ and $y$. The set $W$ is a resolving set for $G$ if distinct vertices of $G$ have distinct codes with respect to $W$. Equivalently, for each pair of distinct vertices

$u, v \in V(G)$ there is a vertex $w \in W$ such that $d(u, w) \neq d(v, w)$. The minimum cardinality of a re-

*This research is supported by The Major Research Project-No. F. 38120/2009(SR) of the University Grants Commission, New Delhi, India. solving set for $G$ is called the resolving number or dimension and is denoted by $\operatorname{dim}(G)$.

\section{An Overview of the Paper}

The concept of resolvability in graphs has previously appeared in literature. Slater $[4,5]$ introduced this concept, under the name locating sets, motivated by its application to the placement of a minimum number of sonar detecting devices in a network so that the position of every vertex in the network can be uniquely determined in terms of its distance from the set of devices. He referred to a minimum resolving set as a reference set and called the cardinality of a minimum resolving set as the location number. Independently, Harary and Melter [3] discovered this concept, but used the term metric dimension, rather than location number. Later, Khuller et al. [2] also discovered these concepts independently and used the term metric dimension. These concepts were rediscovered by Chartrand et al. [6] and also by Johnson [7] while attempting to develop a capability of large datasets of chemical graphs.

It was noted in [8] that determining the metric dimension of a graph is $N P$-complete. It has been proved that the metric dimension problem is NP-hard [2] for general graphs. Manuel et al. [9] have shown that the problem remains $N P$-complete for bipartite graphs. There are many applications of resolving sets to problems of 
network discovery and verification [1], pattern recognition, image processing and robot navigation [2], geometrical routing protocols [10], connected joins in graphs [11] and coin weighing problems [12]. This problem has been studied for trees, multi-dimensional grids [2], Petersen graphs [13], torus networks [14], Benes networks [9], honeycomb networks [15], enhanced hypercubes [16] and Illiac networks [17].

Many resolving parameters are formed by combining resolving property with another common graph-theoretic property such as being connected, independent, or acyclic. The generic nature of conditional resolvability in graphs provides various ways of defining new resolving parameters by considering different conditions. In general, a connected graph $G$ can have many resolving sets. It is interesting to study those resolving set whose vertices are located close to one another. A resolving set $W$ of $G$ is connected if the subgraph induced by $W$ is a nontrivial connected subgraph of $G$. The minimum cardinality of a connected resolving set is called connected resolving number and it is denoted by $\operatorname{cr}(G)$ [18]. In this paper we introduce a new resolving parameter called star resolving number. A resolving set $W$ is said to be a star resolving set if the subgraph induced by $W$ is a star and a path resolving set [19] if $W$ induces a path. In this paper we show the existence of star and path resolving sets in hypercube networks.

\section{Topological Properties of Hypercube Networks}

The hypercube is a very popular, versatile and vertextransitive interconnection network. When the dimension of hypercube increases, the cardinality of its vertex set increases exponentially. The effectiveness of parallel computers is often determined by its communication network. The interconnection network is an important component of a parallel processing system. A good interconnection network should have less topological network cost and meanwhile keep the network diameter as shorter as possible [20].

Definition 3.1. Let $Q^{r}$ denote the graph of $r-d i-$ mensional hypercube, $r \geq 1$. The vertex set

$V\left(Q^{r}\right)=\left\{\left(x_{0} x_{1} \cdots x_{r-1}\right): x_{i}=0\right.$ or 1$\} \quad$ Two vertices $\left(x_{0} x \cdots x_{r-1}\right)$ and $\left(y_{0} y_{1} \cdots y_{r-1}\right)$ are adjacent if and only if they differ exactly in one position. See Figure 1.

The hypercube $Q^{r}$ has $2^{r}$ vertices and $r 2^{r}$ edges. It is $r$-regular and its diameter is $r$. Further it is bipartite, Hamiltonian if $r \neq 1$ and Eulerian if $r$ is even [21]. It has been proved in [22] that $\operatorname{dim}\left(Q^{r}\right) \leq r$. The bound is tight for $r \leq 4$, and it is not tight for $r=5$. A laborious calculation verifies that $Q^{5}$ is resolved by the 4-vertex set $\{00000,00011,00101,01001\}$. Caceres $a: \int_{\substack{1 \\ 0 \\ Q^{1}}}$

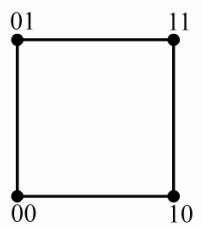

$Q^{2}$

$b: \int_{0}^{1}$

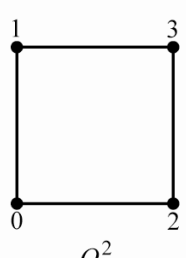

$Q^{2}$

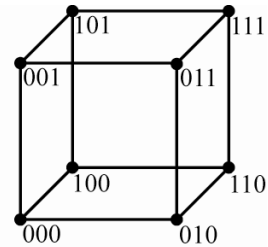

$Q^{3}$

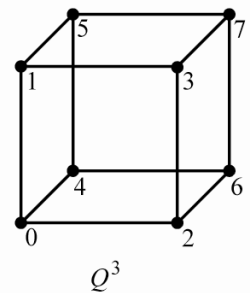

Figure 1. (a) Binary representation; (b) Decimal representation.

et al. [22] have determined $\operatorname{dim}\left(Q^{r}\right)$ for small values of $r$ by computer search; the values are shown in the following table:

$$
\begin{array}{cccccccccc}
r & 2 & 3 & 4 & 5 & 6 & 7 & 8 & 9 & 10 \\
\operatorname{dim}\left(Q^{r}\right) & 2 & 3 & 4 & 4 & 5 & 6 & 6 & 7 & 7
\end{array}
$$

\section{Star Resolving Number}

We begin this section by defining a star and a star resolving set.

Definition 4.1. An $r$-dimensional star, denoted by $S_{r}$ is a graph with one vertex of degree $r-1$ and $r-1$ vertices of degree 1 . The vertex of degree $r-1$ is called the hub of $S_{r}$.

Definition 4.2. A set $W \subset V$ is said to be a star resolving set if $W$ resolves $G$ and if it induces a star. The minimum cardinality of $W$ is called the star resolving number and is denoted by $\operatorname{sr}(G)$.

Remark 1. It is clear that $1 \leq \operatorname{sr}(G) \leq \Delta(G)+1$ for any graph $G$. In a star resolving set the maximum distance between any two locations (vertices) is 2 .

We now proceed to identify a star resolving set in a hypercube network $Q^{r}$. It is clear that there are four copies of $Q^{r-2}$ in $Q^{r}$. We denote them as $Q_{0}^{r-2}$, $Q_{1,1}^{r-2}, Q_{1,2}^{r-2}$ and $Q_{2}^{r-2}$. Figure 2 exhibits the four copies of $Q^{3}$ in $Q^{5}$.

Let $x \in V\left(Q_{0}^{r-2}\right)$. A vertex $x^{\prime} \in V\left(Q_{1,1}^{r-2}\right)$ or $V\left(Q_{1,2}^{r-2}\right)$ is called the image of $x$ if $d\left(x, x^{\prime}\right)=1$. Note that vertices in $Q_{0}^{r-2}$, at distance 1 from $x$ are not considered as images of $x$. If $x^{\prime}$ is the image of $x$ in $Q_{1,1}^{r-2}$ then $x$ is called the pre-image of $x^{\prime}$.

The next result which we state as Lemma 1 is crucial to our work. We omit the proof as this result has been proved in [16] for enhanced hypercubes. 


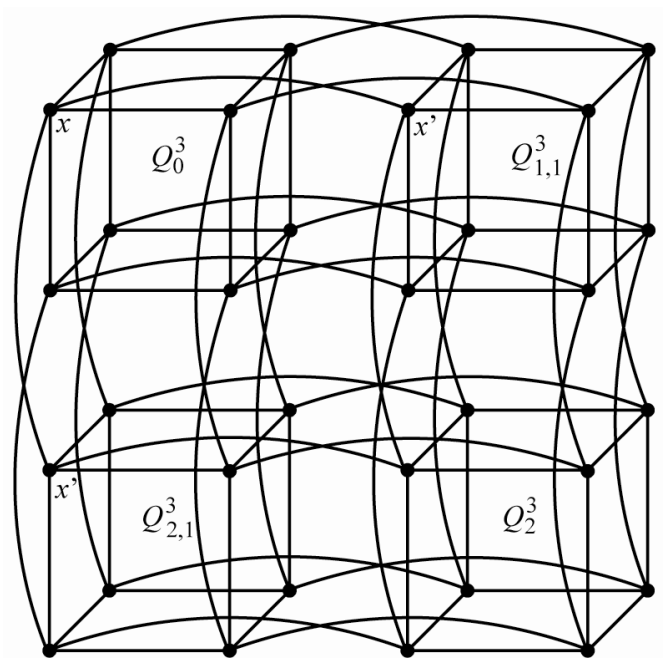

Figure 2. Four copies of $Q_{3}$ in $Q_{5}$.

Lemma 4.1. Let $x \in V\left(Q_{0}^{r-2}\right)$ and let $x^{\prime} \in V\left(Q_{1,1}^{r-2}\right)$ be the image of $x$. Let $w$ be any vertex in $Q_{0}^{r-2}$. Then $d\left(x^{\prime}, w\right)=1+d(x, w)$.

Lemma 4.2. Let $x \in V\left(Q_{0}^{r-2}\right)$. Let $x_{1}^{\prime} \in V\left(Q_{1,1}^{r-2}\right)$ and $x_{2}^{\prime} \in V\left(Q_{1,2}^{r-2}\right)$ be the images of $x$. Then $x_{1}^{\prime}$ and $x_{2}^{\prime}$ are equidistant from every vertex of $Q_{0}^{r-2}$.

Proof. Since the shortest paths from $x_{1}^{\prime}$ and $x_{2}^{\prime}$ to any vertex of $Q_{0}^{r-2}$ pass through $x$, the conclusion follows.

Lemma 4.3. Let $G=Q^{r}, \quad r \geq 1$. Then $\operatorname{sr}(G) \geq r$.

Proof. The subcube $Q_{0}^{r-2}$ of $Q^{r}$ is $r-2$ regular and hence it contains $S_{r-1}$. Now there exist vertices $x \in Q_{1,1}^{r-2}$ and $y \in Q_{1,2}^{r-2}$ such that $x, y$ are equidistant from every vertex of $Q_{0}^{r-2}$ and in particular from every vertex of $S_{r-1}$. This implies $s r(G)>r-1$.

Lemma 4.4. Let $G=Q^{r}$. Then $\operatorname{sr}(G) \leq r$.

Proof. We prove the theorem by induction on $r$.

Base Case: Let $G=Q^{3}$ and $W_{1}=\left\{w_{0}, w_{1}, w_{2}\right\}$, where $w_{0}=0, w_{1}=1$ and $w_{2}=2$. It follows from the definition of hypercube edges that $w_{0}$ is adjacent to both $W_{1}$ and $w_{2}$. It is easy to check that $W_{1}$ is a resolving set for $Q^{3}$. Figure 3 shows the distinct codes of vertices in $Q^{3}$, with respect to $W_{1}=\left\{w_{0}, w_{1}, w_{2}\right\}$. Since $W_{1}$ induces $S_{3}$, it is a star resolving set for $G$.

Now assume that the result is true for the hypercube $Q^{r-1}$. Let $W_{1}=\left\{w_{0}\right\} \cup\left\{w_{i}: 1 \leq i \leq r-2\right\}$, where $w_{i}=2^{i-1}$, be a star resolving set for $Q^{r-1}$. Here $w_{0}$ is the hub and it is adjacent to all $w_{i}, 1 \leq i \leq r-2$. Moreover $W_{1} \subset V\left(Q_{0}^{r-2}\right)$. Divide $Q^{r}$ into four copies $Q_{0}^{r-2}, Q_{1,1}^{r-2}, Q_{1,2}^{r-2}$ and $Q_{2}^{r-2}$. There exist vertices $x \in Q_{1,1}^{r-2}$ and $y \in Q_{1,2}^{r-2}$ having the same codes with respect to every vertex of $Q_{0}^{r-2}$ and in particular with respect to every vertex of $W_{1}$. Hence $W_{1}$ cannot resolve $x$ and $y$. We exhibit a resolving set for $Q^{r}$. Define $W=W_{1} \cup\left\{w_{r-1}\right\}$ where $w_{r-1}=2^{r-2}$ is a vertex

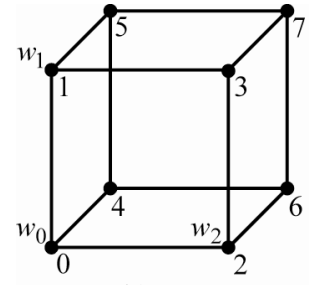

(a)

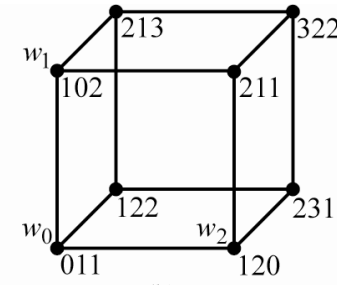

(b)
Figure 3. (a) Resolvingset $W_{1}$ in $Q_{3}$; (b) Codes of vertices of $Q_{3}$ with respect to $W_{1}$.

either in $Q_{1,1}^{r-2}$ or $Q_{1,2}^{r-2}$. Clearly $w_{r-1}$ is adjacent to $w_{0}$. We claim that $W$ is a resolving set for $Q^{r}$.

Case 1: $x, y \in V\left(Q_{0}^{r-2}\right)$ or $V\left(Q_{1,1}^{r-2}\right)$ or $V\left(Q_{1,2}^{r-2}\right)$

Since $W_{1} \subset V\left(Q_{0}^{r-2}\right)$ and since $Q_{0}^{r-2} \cup Q_{1,1}^{r-2}$ and $Q_{0}^{r-2} \cup Q_{1,2}^{r-2}$ are isomorphic to $Q^{r-1}$, by induction hypothesis $W_{1}$ resolves $x$ and $y$. The same argument applies to the following cases.

1) $x \in V\left(Q_{0}^{r-2}\right)$ and $y \in V\left(Q_{1,1}^{r-2}\right)$

2) $x \in V\left(Q_{0}^{r-2}\right)$ and $y \in V\left(Q_{1,2}^{r-2}\right)$

Case 2: $x \in V\left(Q_{1,1}^{r-2}\right)$ and $y \in V\left(Q_{1,2}^{r-2}\right)$

We need to prove that $d(x, w) \neq d(y, w)$ for some $w$ in $W=\left\{w_{0}, w_{1}, w_{2} \cdots w_{r-2}\right\} \cup\left\{w_{r-1}\right\}$. Let $x^{\prime}, y^{\prime} \in V\left(Q_{0}^{r-2}\right)$ be the images of $x$ and $y$ respectively.

Case 2.1: $x^{\prime} \neq y^{\prime}$

In this case

$$
\begin{aligned}
d\left(y, w_{r-1}\right) & =d\left(y, y^{\prime}\right)+d\left(y^{\prime}, w_{r-1}\right)=1+d\left(x^{\prime}, w_{r-1}\right) \\
& =1+1+d\left(x, w_{r-1}\right) \neq d\left(x, w_{r-1}\right) .
\end{aligned}
$$

Case 2.2: $x^{\prime} \neq y^{\prime}$

Now $x^{\prime}$ and $y^{\prime}$ are resolved by some $w$ in $W_{1}$. Hence $d\left(x^{\prime}, w\right) \neq d\left(y^{\prime}, w\right)$ and consequently $d(x, w) \neq d(y, w)$.

Case 3: $x \in V\left(Q_{2}^{r-2}\right)$ and $y \in V\left(Q_{2}^{r-2}\right)$

The proof is similar to Case 2 .

Case 4: $x, y \in V\left(Q_{2}^{r-2}\right)$

Let $x^{\prime}$ and $y^{\prime}$ be the images of $x$ and $y$ respectively. There are three possibilities $x^{\prime}, y^{\prime} \in V\left(Q_{1,1}^{r-2}\right)$ or $V\left(Q_{1,2}^{r-2}\right)$ or $x^{\prime} \in V\left(Q_{1,1}^{r-2}\right)$ and $y^{\prime} \in V\left(Q_{1,2}^{r-2}\right)$. The conclusion will follow by Case 1 and Case 2.

Case 5: $x \in V\left(Q_{1,1}^{r-2}\right)$ and $y \in V\left(Q_{2}^{r-2}\right)$ 
Let $x^{\prime} \in V\left(Q_{0}^{r-2}\right)$ and $y^{\prime} \in V\left(Q_{1,2}^{r-2}\right)$ be the images of $x$ and $y$ respectively. Since $Q_{0}^{r-2} \cup Q_{1,2}^{r-2}$ is resolved by $W_{1}$, there exist a $w \in W_{1}$ such that $d\left(x^{\prime}, w\right) \neq d\left(y^{\prime}, w\right)$. This implies that $d(x, w) \neq d(y, w)$.

Lemmas 3 and 4 imply the following result.

Theorem 4.1. Let $G=Q^{r}, r \geq 1$. Then $\operatorname{sr}(G)=r$.

\section{Path Resolving Number}

In this section we determine a path resolving number for hypercube networks.

Definition 5.1. [19] A resolving set $W$ of $G$ is a path resolving set for $G$ if the graph induced by $W$ is a path. The minimum cardinality of $W$ is called path resolving number and is denoted by $\operatorname{pr}(G)$.

Lemma 5.1. Let $G=Q^{r}, \quad r \geq 1$. Then $\operatorname{pr}(G) \geq r$.

Proof. Let $P$ be the path in $Q_{0}^{r-2}$. Now $P$ cannot resolve $Q^{r}$ as there are vertices $x \in Q_{1,1}^{r-2}$ and $y \in Q_{1,2}^{r-2}$ such that they are equidistant from every vertex of $Q_{0}^{r-2}$ in particular from every vertex of $P$ Since there exist a path $P_{r}$ in $Q_{0}^{r-2}, \operatorname{pr}(G) \geq r$.

Lemma 5.2. Let $G=Q^{r}, \quad r \geq 1$. Then $\operatorname{pr}(G) \leq r$.

Proof. Proceeding as in Lemma 4 we conclude that $W=\left\{2^{i}-1,1 \leq i \leq r\right\}$ is a path resolving set for $Q^{r}$.

Lemma 5 and Lemma 6 imply the following result.

Theorem 5.1. Let $G=Q^{r}, r \geq 1$. Then $\operatorname{pr}(G)=r$.

\section{Conclusion}

In this paper we have introduced a new resolving parameter called a star resolving number. We have determined the star resolving number and path resolving number for hypercube networks. The problem is open for architectures like Benes and Butterfly networks.

\section{REFERENCES}

[1] Z. Beerliova, F. Eberhard, T. Erlebach, A. Hall, M. Hoffman and M. Mihalák, "Network Discovery and Verification," IEEE Journal on Selected Areas in Communications, Vol. 24, No. 12, 2006, pp. 2168-2181. doi:10.1109/JSAC.2006.884015

[2] S. Khuller, B. Ragavachari and A. Rosenfield, "Landmarks in Graphs,” Discrete Applied Mathematics, Vol. 70, No. 3, 1996, pp. 217-229. doi:10.1016/0166-218X(95)00106-2

[3] F. Harary and R. A. Melter, "On the Metric Dimension of a Graph,” Ars Combinatoria, Vol. 2, 1976, pp. 191-195.

[4] P. J. Slater, "Leaves of Trees," Congressus Numerantium, Vol. 14, 1975, pp. 549-559.

[5] P. J. Slater, "Dominating and Reference Sets in a Graph," Journal of Mathematical and Physical Sciences, Vol. 22, No. 4, 1988, pp. 445-455.

[6] G. Chartrand, L. Eroh, M. A. Johnson and O. Oellermann, "Resolvability in Graphs and the Metric Dimension of a
Graph,” Discrete Applied Mathematics, Vol. 105, No. 1-3, 2000, pp. 99-113. doi:10.1016/S0166-218X(00)00198-0

[7] M. A. Johnson, "Structure-Activity Maps for Visualizing the Graph Variables Arising in Drug Design,” Journal of Biopharmaceutical Statistics, Vol. 3, No. 2, 1993, pp. 203-236. doi:10.1080/10543409308835060

[8] M. R. Garey and D. S. Johnson, "Computers and Intractability: A Guide to the Theory of NP-Completeness," Freeman, New York, 1979.

[9] P. Manuel, M. I. Abd-El-Barr, I. Rajasingh and B. Rajan, "An Efficient Representation of Benes Networks and Its Applications," Journal of Discrete Algorithms, Vol. 6, No. 1, 2008, pp. 11-19. doi:10.1016/j.jda.2006.08.003

[10] K. Liu and N. Abu-Ghazaleh, "Virtual Coordinate Backtracking for Void Traversal in Geographic Routing," 5th International Conference on Ad-Hoc Networks and Wireless, Ottawa, 17-19 August 2006.

[11] A. Sebö and E. Tannier, "On Metric Generators of Graphs,” Mathematics of Operations Research, Vol. 29, No. 2, 2004, pp. 383-393. doi:10.1287/moor.1030.0070

[12] S. Söderberg and H. S. Shapiro, “A Combinatory Detection Problem,” American Mathematical Monthly, Vol. 70, No. 10, 1963, pp. 1066-1070. doi:10.2307/2312835

[13] B. Rajan, I. Rajasingh, J. A. Cynthia and P. Manuel, “On Minimum Metric Dimension,” Proceedings of the Indonesia-Japan Conference on Combinatorial Geometry and Graph Theory, Bandung, 13-16 September 2003.

[14] P. Manuel, B. Rajan, I. Rajasingh and M. C. Monica, "Landmarks in Torus Networks," Journal of Discrete Mathematical Sciences \& Cryptography, Vol. 9, No. 2, 2006, pp. 263-271.

[15] P. Manuel, B. Rajan, I. Rajasingh and M. C. Monica, "On Minimum Metric Dimension of Honeycomb Networks," Journal of Discrete Algorithms, Vol. 6, No. 1, 2008, pp. 20-27. doi:10.1016/j.jda.2006.09.002

[16] B. Rajan, I. Rajasingh, M. C. Monica and P. Manuel, "Metric Dimension of Enhanced Hypercube Networks," Journal of Combinatorial Mathematics and Combinatorial Computation, Vol. 67, 2008, pp. 5-15.

[17] B. Rajan, I. Rajasingh, P. V. Gopal and M. C. Monica, "Minimum Metric Dimension of Illiac Networks," Ars Combinatoria (accepted for publication).

[18] V. Saenpholphat and P. Zhang, "Conditional Resolvability of Graphs: A Survey,” International Journal of Mathematics and Mathematical Sciences, Vol. 38, 2003, pp. 1997-2017.

[19] B. Rajan, S. K. Thomas and M. C. Monica, "Conditional resolvability of Honeycomb and Hexagonal Networks," Journal of Mathematics in Computer Science, Vol. 5, No. 1, 2011, pp. 89-99. doi:10.1007/s11786-011-0076-3

[20] H. El-Rewini and M. Abd-El-Barr, "Advanced Computer Architecture and Parallel Processing,” John Wiley \& Sons, Inc., Hoboken, 2005.

[21] J. Xu, “Topological Structures and Analysis of Interconnection Networks,” Kluwer Academic Publishers, Dordrecht, 2001.

[22] J. Caceres, C. Hernando, M. Mora, I. M. Pelayo, M. L. 
Puertas, C. Seara and D. R. Wood, "On the Metric Dimension of Cartesian Products of Graphs,” SIAM Journal of Discrete Mathematics, Vol. 21, No. 2, 2007, pp. 423441. $\underline{\text { doi: } 10.1137 / 050641867}$ 